\title{
THE TREATMENT OF DIABETES IN CHILDHOOD*
}

\author{
BY
}

\section{A. LICHTENSTEIN}

(From the Paediatric Clinic of the Caroline Institute, the Crown Princess Louisa's Hospital,
Stockholm)

The treatment of diabetes in children has interested me now for thirty-five years. In the pre-insulin days I saw the sufferings of diabetic children, who were confined to a diet extremely reduced in carbohydrates and containing practically none of the kind of food that children like. And, in spite of these sufferings, they were condemned to an early death.

Then came insulin which made a rich carbohydrate diet possible. One might have expected a rapid change in diabetic management. This, however, was not the case. On the contrary, during the first insulin years one generally attempted to control the disease as long as possible by reducing the carbohydrate intake and withholding insulin until it was impossible to continue treatment with diet alone. Since that time more liberal ideas on the diabetic diet have slowly spread. Gradually the diet has been brought into closer correspondence with that of healthy persons. So far, however, most diabetes specialists have hesitated to take the decisive step of changing over to a quite normal diet. Moreover, ideas on the diets of diabetics are still highly inconsistent. Different types of diet are prescribed with a highly varying content of calories, as well as of protein, fat, and carbohydrate. This was cleverly demonstrated by Barach in a paper read at the annual meeting of the American Diabetes Association in 1944. He compared the allowances per day for diabetic children at different ages by various groups; for instance, by the Medical Research Council in England, by the National Research. Council in the United States, and by the League of Nations Committee. Barach concluded that, depending upon which of these standards one happened to accept, we would prescribe from 20 to 300 per cent. more or less of calories, and the same lack of agreement holds true for the allowanice of the different constituents of the food. We find, for instance, for protein 100 per cent. variation in the daily allowance. And for whichever standard we might adopt we would have a recognized 1949.

- A paper read at a meeting of the British Paediatric Association, authority in every instance. It is astonishing that these and similar facts have not led to the conclusion that no ground exists for the acceptance of a scientifically founded restricted diet, prescribed in every detail.

\section{Our Present Knowledge of Nutritional Requirements of Diabetes}

It may be useful to review shortly our present knowledge of the diabetic's need of food and of its various constituents. There are no valid reasons for assuming a greater caloric need for the diabetic than for a non-diabetic individual of the same age. Nor has any conclusive evidence been advanced for the supposition that the diabetic child needs more protein than the non-diabetic one. The question of the fat allowance is closely connected with the carbohydrate intake. In the past fat made up a large part of the caloric intake of the diabetic. To-day, however, the need of a well-balanced diet for the formation and decomposition of ketone bodies is generally accepted, and consequently the fat allowance has been reduced to about 20 to 30 per cent. of the total caloric intake, that is, the same as in the normal diet.

About half of the caloric intake of normal adults and children consists of carbohydrates. Because of the nature of diabetic metabolism, the carbohydrates obviously had to be greatly reduced in the pre-insulin era. With the introduction of insulin a more liberal carbohydrate allowance became possible. To-day, 40 to 50 per cent. of the caloric intake of many diabetics is composed of carbohydrates, although many physicians still cling to a more restricted carbohydrate intake.

In estimating the carbohydrate allowance for a diabetic it is necessary to remember first that the diabetic organism is deficient in glycogen and has to struggle against a dysfunction in the formation and deposition of glycogen in the liver, and secondly that the carbohydrates are, in Macleod's phrase, the fuel of life even for the diabetic. A sufficient carbohydrate intake, made possible through insulin, is and must be the cornerstone in the treatment of a diabetic. Long ago we also learned from Allen and Dubois that a diabetic utilizes the carbohydrates in spite of hyperglycaemia and glycosuria, and this has been confirmed in many quarters. 
The knowledge that sugar is formed both from protein and fat has thrown a new'light on the question of the intake of the various food constituents. Geelmuyden states that all three, protein, fat, and carbohydrate, mingle inseparably in the intermediary metabolism, react mutually, and form new combinations, and that it is impossible to determine which of them is the primary source of the new-built sugar. Soskin and Levine also point out that 'in the light of more recent knowledge of intermediary metabolism, it seems likely that we shall soon cease to distinguish between the metabolisms of the different foodstuffs, once they have gone beyond certain stages, for eventually all of them give rise to very similar intermediary products, namely the $\alpha$ - and $\beta$-keto acids,' and they speak of ' a final common pathway for all the foodstuffs.'

Our present knowledge of the importance of vitamins for the diabetic can be summarized as follows.

There is no evidence of a lack of vitamin $A$ in diabetics. At least three elements of the vitamin B complex are of importance for the carbohydrate metabolism. Thiamine pyrophosphate is the co-enzyme necessary for the oxidation of pyruvic acid. The yellow enzyme, necessary in hexose oxidation, is now known to be a riboflavin phosphate and protein combination. One of the co-enzymes involved is nicotinic acid. Despite the important role of the vitamin $B$ complex in carbohydrate metabolism it seems evident that the diabetic generally receives so much from the food that surplus amounts are of no importance in the treatment of diabetes. My observations agree with Joslin's that no notable effect on the blood sugar or insulin requirement can be observed, even when large doses are given.

Patients with uncomplicated diabetes and adequate vitamin $\mathrm{C}$ intake have normal plasma levels of ascorbic acid. Hamne found that the injection of 200 . to $500 \mathrm{mg}$. of ascorbic acid, intravenously, somewhat intensified the action of insulin on the blood sugar level. Owens et al., however, gave diabetics 300,600 , and $1,200 \mathrm{mg}$. of ascorbic acid daily without noting any constant improvement My own experience points in the same direction. It would seem, therefore, that diabetics, provided their intake is adequate, have no greater need of vitamin $C$ than normal individuals. Nothing of special importance is known of the diabetic's requirements of vitamins $D, E$, and $K$.

While the importance of the mineral salts in diabetes is unquestioned, a mixed diet of natural foods which is adequate in other respects will also provide a sufficient amount of the minerals.

To sum up: it should be emphasized that in recent years there has been increasing agreement that the diabetic needs an adequate, normal, well balanced diet with an ample supply of vitamins, that is, a diet based on modern knowledge of the physiology of nutrition.
Experiments with 'Free Diets'

It was such considerations as these which led some paediatricians some twenty years ago to raise the question: Does a proper control of diabetes in children require a strictly regulated diet or is it possible to control the disease with insulin only, without dietetic restrictions?

It is not a mere coincidence that the treatment of diabetes without dietetic restrictions has been taken up by paediatricians. Diabetes in children is essentially different from diabetes in adults, and the difficulties incidental to a more or less rigorous diet make themselves much more strongly felt in children than in adults.

In children, diabetes is almost always severe. It is, moreover, characterized by a more labile metabolism than in adults, with sudden oscillations between hyperglycaemia and hypoglycaemia and with a perpetual menace of ketosis. Children are also more liable to infection. Furthermore, diabetic children require an adequate supply of food for growth and development, whereas adult diabetics need only be maintained in equilibrium and can even periodically be kept with advantage on a somewhat low diet.

Last but not least we must take into account the psychological factors. The maintenance of a diet which is to be observed not for weeks or months but for life is harmful to mental development, character formation, and social adjustment. There is no doubt that the diabetic regimen produces behaviour difficulties in children. But we want the diabetic child to develop in a normal way not only physically but also mentally. We should therefore, as far as possible, avoid placing these children in a position apart from other children, and I see in this an important reason in favour of the 'free diet.'

The first experiences in the treatment of diabetes in children without any dietetic restrictions were published in 1931 by Stolte in Breslau. In 1933 Söderling in Stockholm reported a number of good results obtained through the same method. In 1932 I tried the effect of the 'free diet' on a minor scale. In fact one patient of mine has had the 'free diet' since 1924. He is still living in good general health and without any signs of arteriosclerosis. Since 1933 I have consistently carried out treatment with the 'free diet.' I mean by this term a normal diet corresponding completely with that of healthy children. The diabetic children are allowed to share the diet of their brothers and sisters, to eat their fill and to satisfy their individual tastes within the same limits as healthy children. Even sugar and sweets, in reasonable amounts, are permitted. The only restriction is avoidance of over-indulgence, just as one would impose on any child. The " free diet 'is thus a normal, balanced, all-round fare, which is not the case with high-fat, high-protein, and high-carbohydrate diets.

The amounts of food which the diabetic children spontaneously consume in the hospital are weighed and measured. As a rule, the children take 150 to 
TARE 1

DAILY FOOD INTAKE OF DIABETIC CHILDREN

\begin{tabular}{|c|c|c|c|c|c|c|c|c|c|c|c|c|}
\hline \multirow[t]{2}{*}{ Age (years) } & \multicolumn{3}{|c|}{ Barach, 1944} & \multicolumn{4}{|c|}{ Collens and Boas, 1946} & \multicolumn{2}{|c|}{$i$} & \multicolumn{2}{|c|}{ ' Free Diet ' } & \multirow[b]{2}{*}{ Fat } \\
\hline & Calories & $\begin{array}{l}\text { "Carbo- } \\
\text { hydrate }\end{array}$ & Protein & Fat & Calories & $\begin{array}{l}\text { Carbo- } \\
\text { hydrate }\end{array}$ & Protein & Fat & Calories & $\begin{array}{l}\text { Carbo- } \\
\text { hydrate }\end{array}$ & Protein & \\
\hline $1-3$ & $85-110$ & $10-14$ & $3-4$ & $3 \cdot 5-4$ & $90-100$ & $11-12$ & $3-4$ & $3-4$ & $90-100$ & $10-11$ & & \\
\hline $4-6$ & $78-80$ & 10 & 3 & $3 \cdot 2-3 \cdot 5$ & $80-90$ & $10-11$ & $3-3 \cdot 5$ & $3-3 \cdot 5$ & $80-90$ & & & \\
\hline 7-9 & $64-72$ & $8-9$ & $2 \cdot 5-3$ & $2 \cdot 6-3$ & $70-80$ & $9-10$ & $2 \cdot 5-3$ & $2 \cdot 5-3$ & $70-80$ & & & \\
\hline $10-12$ & $56-63$ & $7-8$ & $2 \cdot 0-2 \cdot 3$ & $2-2 \cdot 5$ & $60-70$ & $7-9$ & $2 \cdot 5-3$ & $2 \cdot 5-3$ & $50-70$ & 1 & $2-3$ & $2 \cdot 5-3$ \\
\hline $13-15$ & 44-55 & $6-7$ & $1 \cdot 8-2 \cdot 0$ & 2 & $50-60$ & $7-9$ & $2-2 \cdot 5$ & $2 \cdot 5-3$ & $40-60$ & $i$ & 1 & \\
\hline
\end{tabular}

- All values in rounded $\mathrm{g}$ - per $\mathrm{kg}$. body-weight.

250 g. carbohydrate per day, corresponding to 6 to $7 \mathrm{~g}$. per kilogram of body-weight per day, with relatively small variations from day to day. Younger children consume considerably more, up to 8 to $10 \mathrm{~g}$. or more per kilogram of body-weight per day. Some older children, periodically, also seem to show a relatively large need of carbohydrates. Of protein, the children consume, as a rule, 2 to $3 \mathrm{~g}$., and of fat 2.5 to $3.5 \mathrm{~g}$. per kilogram of body-weight per day.

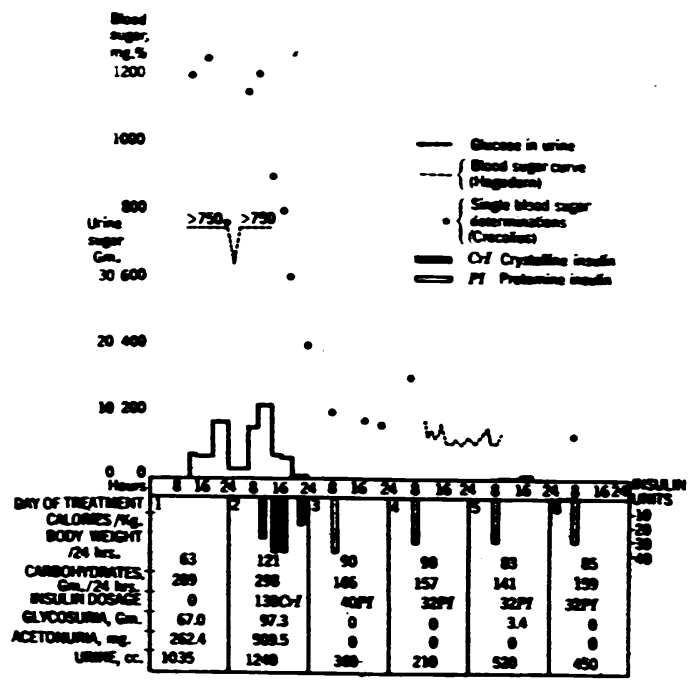

Fig. 1.- Chart showing control in a fresh case.

The supply of calories was as a rule spontaneously maintained at 80 to 100 under 5 years of age, at 60 to 80 between 5 and 10 years, and at 40 to 60 between 10 and 15 years, all per kilogram of bodyweight.

It is of interest to compare the average supply of food on the 'free diet' with the allowances of calories, carbohydrate, protein, and fat in recent diets prescribed for diabetic children. Table 1 shows the allowance in Barach's diet (1944) and in that of Collens and Boas (1946), compared with the spontaneous intake of Swedish children on a "free diet.'

From the table it may be gathered that the amounts of carbohydrates, protein, and fat which are prescribed in diets for diabetic children in part considerably exceed the spontaneous intake of my patients. In fact, there exist prescribed diets with more carbohydrate and less carbohydrate, with more protein and less protein, with more fat and less fat, than the normal diet of healthy Swedish children.

Naturally, my own experience applies only to Swedish children. The figures cited cannot simply

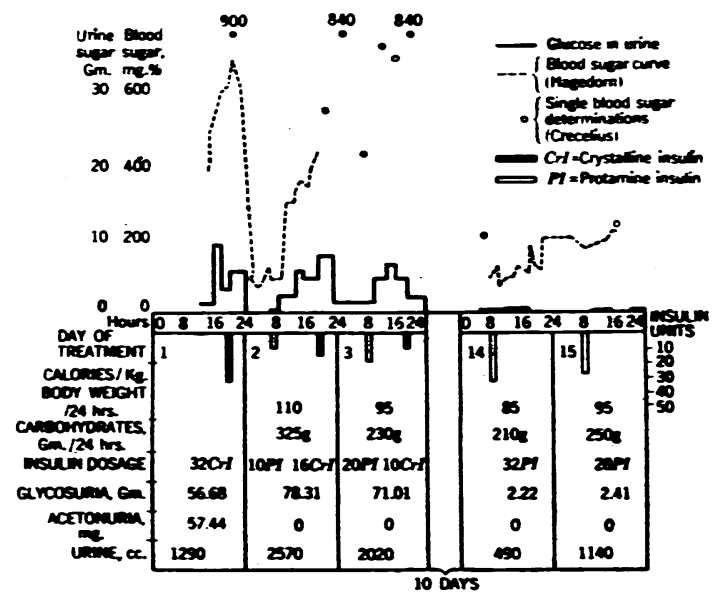

Fig. 2.-A second chart showing control in a fresh case.

be transferred to all countries, in view of the great variations in different parts of the world. Everyone must be aware of the difference in the diet of the Eskimos and the Negroes. However, it is probable that the Swedish figures are applicable in countries where the normal diet is a balanced one, satisfactory from the modern point of view of the physiology of nutrition.

A fresh case of diabetes is always given the ordinary hospital fare. In most cases control is 
obtained within a few days or at most a week, as is shown on figs. 1 and 2 . I consider a patient under control when he has a feeling of well-being, has a normal appetite, normal thirst, a normal quantity of urine, and is free of all subjective symptoms. It has, however, not been my aim to keep the urine sugar-free, provided the child is otherwise symptomfree. I think some tens of grams of sugar per twenty-four hours are permissible. As a rule, the degree of glycosuria is less than 10 per cent. of the carbohydrate intake. It is obvious that there is a slighter risk of hypoglycaemia under such circumstances than with attempts to obtain a completely sugar-free urine. Moreover, I think Lawrence is right when he states that no case of severe diabetes can be kept continuously sugar-free without risk of hypoglycaemia by any present method of insulin treatment which is tolerable to the patient. And I agree with him also when he says that such an ambition may stimulate the diabetic purist but is a curse to his patient. In my patients severe hypoglycaemic attacks are rare. They occur occasionally when there is intercurrent acute disease with loss of appetite or vomiting, or when children are taken out of their normal routine and have meals irregularly, as for example, during a long journey.

Permitting a slight glycosuria I do not aim at ' normalizing' the blood sugar level. One cannot take for granted that what is a normal blood sugar level in a non-diabetic individual is necessarily 'normal' for a diabetic. Actually many authors have stressed the importance of a glucose level in the blood sufficient for the storage of glycogen in the liver. In reality, one should adhere to the aim that the diabetic utilizes carbohydrates as completely as possible in comparison with his need; in other words, that the carbohydrate balance, the difference between intake and excretion, should be as great as possible. It must always be remembered that it is utilization and not excretion that is important.

Of course, it is not at all difficult to depress the blood sugar level; it is only necessary to reduce the quantity of food, but that in no way implies that the utilization of carbohydrates has improved. On the contrary, we know that starvation, as well as a diet high in fat in comparison with the carbohydrates, will lead to fatty infiltration of the liver. The blood sugar may then be 'normalized'; however, 'the diabetes is better, but the patient is worse.'

In my opinion, when the increase in the blood sugar level is limited, no attempt need be made to bring the level down to normal. This should not be taken to mean that the blood sugar may be allowed to rise to many hundred milligrams per $100 \mathrm{ml}$. In well controlled cases on the 'free diet' the fasting values of the blood sugar usually range between 100 and $200 \mathrm{mg}$. per $100 \mathrm{ml}$. The twentyfour-hour curve often shows considerable variations, but that is also true of diabetic children on a restricted diet.

Non-normalization of the blood sugar has caused no difficulty in any of our cases. Assuming that the rise in blood sugar is moderate, there is no difficulty in keeping the patient symptom-free in other respects. An unfavourable effect of a somewhat higher blood sugar was not demonstrable on either the frequency and course of infections or the frequency of complications.

Although a small or moderate excretion of sugar is permitted in our patients, ketonuria is not. In our opinion, scrupulous attention to this plays a decisive role in the favourable course of diabetes treated by 'free diet.' As Bertram, among others, points out, it is the ketosis and not the blood sugar level which is responsible for a severe course of the disease. It was found that the 'free diet' greatly aided our efforts to keep patients free of ketosis, and that a previously marked tendency towards ketosis frequently disappeared when patients were transferred to it. Children with constant signs of ketonuria, who in many cases were repeatedly sent to hospital because of menacing or full-blown coma, after removal of all dietetic restrictions for years showed no trace of ketonuria. Compared with our previous experience, the frequency of pre-coma and coma was markedly reduced by the 'free diet.'

Now, in the light of my experience, I think it is established that diabetic children on the 'free diet ' grow and develop in a normal way, pass through puberty normally, are able to work physically and mentally, and show a considerably stronger resistance to acute infections, as well as to tuberculosis, than do patients treated with a diet restricted in carbohydrates. The former also show a considerably lower frequency both of coma and of hypoglycaemic attacks. In each of these respects, treatment with insulin and without any dietetic restrictions is undoubtedly at least as valuable, and, from certain points of view, probably superior to treatment with diet plus insulin.

\section{The 'Free Diet' and the Frequency of Complications}

Evaluation of any method of treating diabetes requires, however, not only consideration of these circumstances but also of the frequency of various complications. Many authors have in the last years reported an alarming frequency of cardiovascular and ocular complications in juvenile diabetic patients who have been treated for ten to twenty or more years. Particularly remarkable are White's figures of known vascular complications in 203 (or 92 per cent.) of 220 cases (table 2). These

TABLE 2

VASCULAR DISEASE IN 220 CASES OF JUVENILE DIABETES SURVIVING TWENTY YEARS OR MORE OF THE DISEASE (WHITE)

Retinal arteriosclerosis

Per cent.

Retinal haemorrhages

Calcified arteries

Hypertension

Albuminuria

Coronary insufficiency

Cerebral vascular accidents 
are indeed frightening, especially when one considers that the source is perhaps the best organized institution for the treatment and control of diabetes with a restricted and carefully controlled diet. White, it is true, says that 'these are the worst possible statistics which will ever be presented since these patients did not receive modern treatment for 50 per cent. of their diabetic lives.' But insulin treatment has been used for twenty-five years now. Dolger's figures are even worse; in 200 regularly examined patients he found' ' no single instance where the diabetes had lasted twenty-five years in which the patient escaped the development of vascular damage.' It seems not improper to point out that a method of treatment giving such a high degree of severe complications is in great need of improvement.

A priori, it is not very probable that treatment with the 'free diet' will prove to be considerably superior to treatment by diet, in so far as frequency of complications is concerned. In fact, the differences between prescribed diets and the "free diet" often are insignificant, even though a low-carbohydrate diet is still often prescribed. We need new discoveries. Perhaps the newer knowledge of the pituitary and adrenal hormones may lead to as yet untouched means of therapy. One can only hope that work such as that of the Coris, of Houssay, and of their followers will finally give the chinicians better weapons for preventing complications.

However, the actual question here is whether the frequency of complications on the "free diet' is higher, lower, or equal to the complications which arise on the restricted diets. From this point of view, proof that the frequency of complications is not higher on the 'free diet' should suffice. The possibility that the 'free diet' will give a reduced frequency of complications is not excluded, but it is too early for a definite statement in this matter.

What do we know about the causative factors of the cardiovascular complications? It would entail too long a discussion to take up the whole problem, so I must limit myself to a few points. First, it may be stated that the arteriosclerotic lesions complicating juvenile diabetes on the whole are identical with those seen in persons of advanced years. The assumption is therefore justified that the etiology, at least in part, is the same. Even reckoning with the many different causes contributing to the development of arteriosclerosis, constitutional and hereditary factors, senescence, as well as disturbances in the hormonal balance and toxic and mechanical causes, a great many facts speak in favour of the concept that nutritional causes in the form of a disturbed lipid metabolism and acid-base balance are concerned in the development of cardiovascular lesions in diabetes. We know that it is possible to produce arteriosclerosis of the human type in animals by feeding them cholesterol. We also know that increase in blood cholesterol is a regular phenomenon in arteriosclerosis in man. Now, poorty controlled or uncontrolled diabetes is often associated with a considerable increase of blood cholesterol and the cholesterol level rises with the severity of the disease. It thus seems obvious that there is a close relation between cardiovascular disease in diabetes and hypercholesteraemia.

Ketosis seems also to be important in the development of arteriosclerosis. We know that a ketogenic diet provokes a high blood pressure in rabbits and that the changes are more severe in animals in which the acid-base balance is much disturbed. The blood fat varies in diabetes with the acidosis. It may therefore be supposed that a ketogenic diet plays a role in the development of arteriosclerosis in juvenile diabetes. As already pointed out the frequency of coma was reduced by the 'free diet.' On the other hand the assumption that there is some connexion between high blood sugar levels and the appearance of cardiovascular lesions has not been confirmed.

From these points of view, it seems reasonable that thecarbohydrate-rich 'free diet ' may be of some value in preventing or postponing juvenile arteriosclerosis. In any case, nothing favours the opinion that a diet poor in carbohydrates, that is, to a certain extent a ketogenic diet, is preferable in this respect.

In my report in 1945 on ten years' experience with the "free diet' I had to describe few complications. Since then I have had the same experience as Priscilla White, Dolger and others that vascular complications become more and more frequent after the tenth year of diabetes.

Our own cases are now being systematically re-examined for complications. The data are still incomplete. To-day I am only able to give preliminary figures on about fifty cases with a history of diabetes of over twelve years. We have found albuminuria in about 30 per cent., hypertension also in about 30 per cent., calcified arteries in about 14 per cent., and retinal haemorrhages or other pathological findings in the eyes in about 60 per cent. However, in most cases with retinal haemorrhages there were no subjective symptoms. Only in about 13 per cent. could a considerable visual reduction be demonstrated.

So far it seems that vascular eye complications in my patients occur with about the same frequency as in patients treated with a restricted diet, but that hypertension, and particularly calcification in the peripheral arteries, occur somewhat less frequently.

The high frequency of vascular damage is, however, appalling, and I agree with Priscilla White that vascular disease in diabetes must be considered a challenging problem for future solution. To-day we can only state that the duration of the diabetes is, in Dolger's phrase, "the only unequivocal factor in the determination of the appearance of vascular damage.'

To sum up, I should like to point out that our present knowledge does not justify the assumption that the danger of vascular damage is greater in diabetics on the "free diet 'than in those on restricted diets. If anything, the contrary is probable. 
For the present, however, this is only a hypothesis. A mere ten to fifteen years of observation are an inadequate basis for judging the frequency of diabetic complications, and with the exception of a

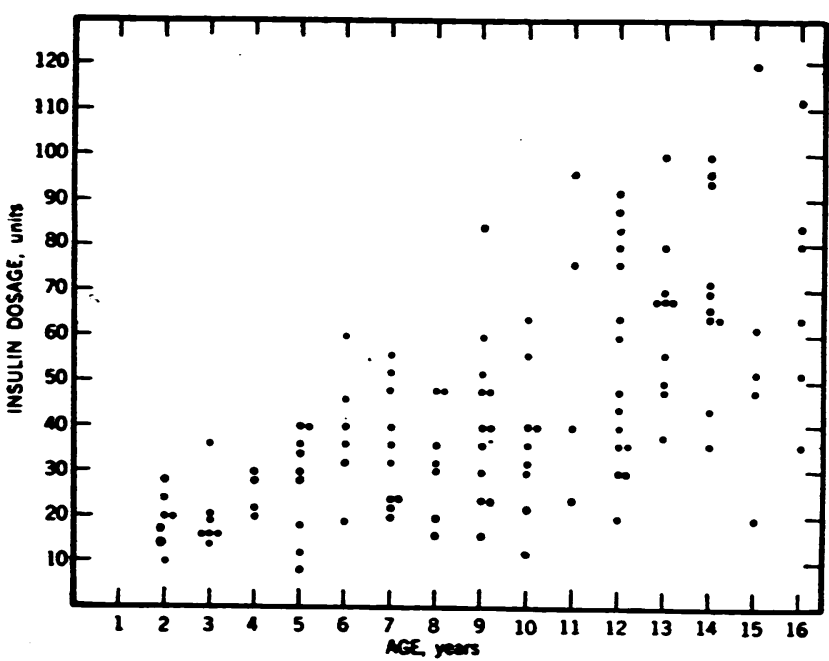

FIG. 3.-Diagram showing the insulin dose during the second year of the 'free diet'.

few cases, treatment with the 'free diet' is not yet twenty years old. In five to ten years it will be possible to be more positive in answering this question. The real problem to-day in judging the value of any method of treating diabetes is its capability of preventing complications.

The mortality rate is, of course, also of great importance. For a number of reasons, however, comparisons between the various rates is particularly difficult in diabetes. Thus, it is possible to draw comparisons only with great reserve, and the best statistics for this purpose are those from one and the same country.

A comparison between published figures with strictly prescribed diets and with the ' free diet' covering a period of fifteen years' treatment shows that the mortality rate of diabetes on the ' free diet' does not suffer by comparison with the results on the restricted diet. Difficult as it is to arrive at exact comparisons, it yet seems obvious that the mortality rate of our patients on the 'free diet' for fifteen years, about 6 per cent., is not higher and is probably lower than the best results achieved with restricted diets.

The overall picture of fifteen years' treatment of diabetes in children without dietetic restrictions is thus a very satisfactory one: good physical and mental development, a minimum of coma and hypoglycaemia, a frequency of complications not higher and possibly lower than in treatment with restricted diets, and a low mortality.
Conditions of Seccess Using the 'Free Diet'

The essential conditions for satisfactory results with the 'free diet' are, first, adequate insulin treatment from the outset, and second, careful continuous control.

Insulin supply. An adequate insulin supply is the prerequisite for every satisfactory diet for diabetic children. The first consideration is the choice of the preparation. Previously, treatment without dietetic restrictions required two, and often three, doses of ordinary insulin a day. Now that we have obtained new insulin preparations with a slow absorption time, treatment has been simplified. I have used Hagedorn's protamine insulin with great advantage, and have been able in most cases to reduce the number of injections to one a day. As a rule, it is given in the morning, about half an hour before the first meal. In a small number of cases, an evening dose is also required. Sometimes it is of advantage to combine protamine with a dose of crystalline insulin at the same time. No account has been taken of the endogenous liver rhythm, nor was there any necessity for this in my material. In most cases, zinc protamine insulin was found to be less beneficial for children. On this insulin, they showed a tendency to hypoglycaemia more frequently than on the protamine insulin without zinc. Occasionally, however, one finds a child who adjusts himself more easily to the insulin with zinc than without it. In a single case, the old type of

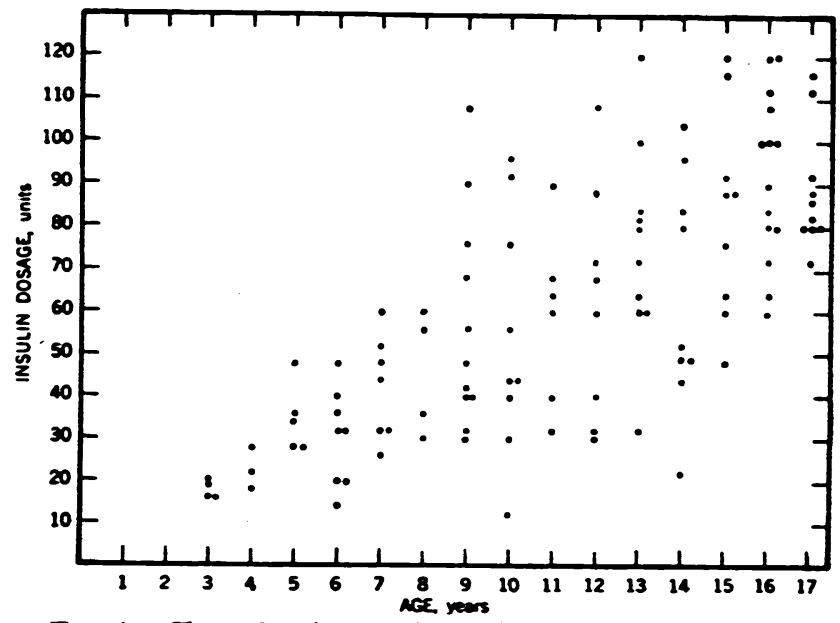

FIG. 4.-Chart showing maximum insulin dose with the

$$
\text { 'free diet'. }
$$

insulin was found to be superior to the protamine type. It is evident that in the choice of preparation proper allowance must be made for individual differences.

The insulin requirement varies greatly from case 
to case, and must be individually tested for each child. Especially in the initial stage of the disease, very careful control and flexibly adjusted changes in the insulin supply are required.

To gauge the insulin requirement of a diabetic child being treated without dietetic restrictions, the insulin dosage should be observed for a sufficiently long time after treatment is instituted, if necessary, during the second year even, by which time practically all cases attain a certain stability in insulin requirement. Fig. 3 shows the relation between the patient's age and the insulin requirement, and the variation in the amount of insulin required; the dosages range from 10 to 120 international units per day, with an average of about $\mathbf{4 0}$ units.

Fig. 4 shows the maximum insulin requirements of our patients at any time during the observation period, attacks of coma excluded. Here, too, the range is between 10 and 120 units, but the average, about 60 units, is of course higher.

Control of the case. Careful, continuous control is essential if good results are to be obtained with a normal diet. Examinations must be frequent at first; gradually, they can be spaced further apart. When the patients are children, such control involves good co-operation with the home, especially with the mother. Mothers must, therefore, be taught the principal features of the clinical treatment of the disease. It is particularly important that mothers learn the significance of increased thirst and large amounts of urine as signs of unsatisfactory adjustment, and the importance, in such cases, of consulting the doctor immediately. Mothers must also learn to recognize the signs of hypoglycaemia and how to counteract it. Stress should be laid on the importance of a regular conduct of life and regular meals.

The 'free diet' has been adopted by many physicians, particularty in the Scandinavian countries and in Germany, and numerous reports have been published on the results in juvenile diabetes as well as in adults. On the basis of the published material, it would seem that those using this treatment are satisfied with the results. Nevertheless, the method has met with violent opposition. This was to be anticipated, since it called for a break with the longfixed concept of the necessity of a restricted diet in the treatment of diabetes.

Various arguments have been used by those objecting to the 'free diet.' I have already dealt with most of these. A further objection has been that the 'free diet' would considerably increase the insulin requirement. As early as 1929 Richardson demonstrated that carbohydrates might be substituted for fat in the diet without increasing the insulin dosage. The amount of insulin required by patients on the 'free diet' has been about the same as by diabetics on a restricted diet. This has been confirmed by a recent study of a large group of diabetic patients in Sweden: the average amount of insulin per day and per individual required by 915 patients of varying ages, who carefully followed a strict prescribed diet, was 52 units, whereas 4,639 patients on the 'free diet' needed an average of 56 units daily. That is an insignificant difference. Patients with a ten-year duration of the disease used an average of 65 units daily, whether they had kept to a restricted diet (256 cases) or were on a normal diet (1,333 cases).

In spite of all opposition, the 'free diet' gains ground every day.' One important reason for this is the difficulty, not to say impossibility, of keeping a patient on a strictly regulated diet for decades. According to Joslin, "breaking the diet and cheating the doctor are infrequent episodes to-day.' But his co-worker, White, states:

'Incorrectly kept (perfect) urine charts, the substitution of water for a specimen of urine, the substitution of an obliging normal friend's urine for the patient's own, while annoying to the physician, should not be considered abnormal behaviour for the juvenile diabetic.'

In the Swedish investigation we found that of 5,207 diabetics of all ages only 35.7 per cent. carefully followed the instructions regarding diet. Thus two-thirds of all patients followed their prescribed diets carelessly or not at all. I doubt if similar investigation in other countries would give a better result. Is it not wiser to give the diabetic freedom to eat the food which is normal for his home, age, and work, and adjust the insulin dosage for control under these circumstances rather than to prescribe a diet which in the long run only one-third of the patients follow and thereby run the risk of insufficient control and perhaps insufficient insulin dosage? True, the insulin requirement of patients on the 'free diet' is on the average not much higher than that of patients on restricted diets. Nevertheless, in the individual case, there may be a difference of 10 per cent. or more, a difference which can be of great importance for the control of the disease.

Many physicians who still prescribe a diet now declare that this diet should be a normal one. The difference between the advocates of the 'free diet' and those who prescribe a normal diet is obviously only the answer to the question: Is it necessary to prescribe a normal diet in the form of a detailed diet chart or is it not sufficient to prescribe a regular conduct of life and avoidance of overfeeding ? I for my part am convinced that a normal diet can be maintained without any other restrictions than the ones just mentioned. 
In my opinion the many objections to the 'free diet' have not stood the test of time. Finally I wish once more to stress that psychological considerations favour the 'free diet.' Liberation of a diabetic child from its position of being different from other children must be beneficial to the child's mental development, character formation, and social adjustment. It is well known that mental strain has an unfavourable effect on diabetes. I believe that a feeling of well-being, on the contrary, has a favourable effect. Is it not time for those who impose restrictions on their patients to show that their results are better than control with insulin alone and without restrictions? 